\title{
MÉTHODE DE MESURE DE LA CONDUCTIBILITÉ THERMIQUE DES MATÉRIAUX RÉFRACTAIRES, BONS CONDUCTEURS ÉLECTRIQUES
}

\author{
par Z. MIHAILOVIC et G. NAUDET $(*)$ \\ Service de Physique du Solide et de Résonance Magnétique \\ Centre d'Etudes Nucléaires de Saclay, B. P. no 2, 91, Gif-sur-Yvette, France
}

(Reçu le 26 juin 1970, révisé le 14 septembre 1970)

\begin{abstract}
Résumé. - On décrit la mise au point d'une méthode absolue pour la mesure de la conductibilité thermique $\lambda$ des matériaux réfractaires, bons conducteurs électriques $\left(\mathrm{ZrO}_{2}+5 \% \mathrm{CaO}\right.$ et $\left.\mathrm{ZrO}_{2}+10 \% \mathrm{Y}_{2} \mathrm{O}_{3}\right)$ à haute température $\left(1000-2400{ }^{\circ} \mathrm{C}\right)$. Cette méthode est fondée sur la différence de température qui apparaît dans le sens radial dans les échantillons de forme cylindrique quand ces derniers, non isolés thermiquement, sont chauffés directement par effet Joule. L'idée de cette méthode vient de celle de la mesure de la conductibilité électrique $\sigma$ utilisant le même mode de chauffage. Un raisonnement mathématique donne l'expression exacte de $\lambda$ et permet de déterminer la température équivalente $T_{\mathrm{e}}$ à laquelle correspondent les valeurs de $\sigma$, que l'on mesure d'ailleurs en même temps que $\lambda$. Comparée aux méthodes classiques, celle que l'on présente ici se montre à la fois très rapide et d'une mise en œuvre facile.
\end{abstract}

Abstract. - An absolute method is described for measuring, at high temperatures (1 000$2400{ }^{\circ} \mathrm{C}$ ), the value of the thermal conductivity $\lambda$ of refractory materials with good electrical conductivity. The method is based on the radial temperature difference set up in a non insulated cylindrical specimen by direct joule heating. The idea is inspired by the method for measuring the electrical conductivity using the same mode of heating. An exact expression is given for $\lambda$ as well as for the equivalent temperature $T_{\mathrm{e}}$ to which corresponds the value of $\sigma$ which is measured simultaneously. The technique presented is both very fast and quickly set up in comparison to the classical methods.

Introduction. - Le développement des nouvelles techniques dans le domaine des hautes températures exige de plus en plus des matériaux de caractéristiques bien définies pouvant résister dans les conditions d'utilisation à l'action simultanée de plusieurs facteurs physiques, chimiques et mécaniques. La conversion directe de l'énergie thermique en l'énergie électrique par la voie magnétohydrodynamique (M. H. D.) est un bon exemple de ces techniques; la réalisation des électrodes destinées à fonctionner dans la veine d'un générateur $M$. H. D. demande en particulier des matériaux réfractaires, bons conducteurs électriques à haute température $\left(1600^{\circ} \mathrm{C}\right.$ au minimum $)$ et d'une bonne tenue mécanique en atmosphère oxydante. La zircone stabilisée, $\mathrm{ZrO}_{2}+\mathrm{CaO}$ ou $\mathrm{ZrO}_{2}+\mathrm{Y}_{2} \mathrm{O}_{3}$ par exemple, a été proposée pour cette application du fait qu'elle a parmi les oxydes réfractaires, en plus de sa bonne tenue en atmosphère oxydante, une conductibilité électrique des plus élevées à haute température $\left(\sim 1 \Omega^{-1} \mathrm{~cm}^{-1}\right.$ à $\left.1800^{\circ} \mathrm{C}\right)$. Ces électrodes doivent en outre assurer l'évacuation d'un flux thermique important qui peut varier de 50 à $400 \mathrm{~W} / \mathrm{cm}^{2}$. Une bonne connaissance de la conductibilité thermique

(*) Actuellement au DB/GEERSM, C. E. N. Saclay. de ces céramiques est, par conséquent, indispensable pour la construction de ces convertisseurs d'énergie. Elle permet de déterminer le refroidissement nécessaire des électrodes.

Nous avons cherché à mettre au point une technique de mesure de la conductibilité thermique $\lambda$ de ces matériaux entre 1100 et $2500^{\circ} \mathrm{C}$ en atmosphère oxydante en faisant appel à la méthode absolue, c'est-à-dire à celle qui ne se réfère pas à un échantillon étalon de conductibilité thermique connue.

La propriété très importante de ces réfractaires, $\mathrm{ZrO}_{2}+\mathrm{CaO}$ et $\mathrm{ZrO}_{2}+\mathrm{Y}_{2} \mathrm{O}_{3}$, de conduire relativement bien le courant électrique dans ce domaine de température, permet d'employer le chauffage direct par effet Joule pour les porter à haute température. Ce moyen de chauffage des échantillons a été déjà utilisé dans les travaux précédents [1] pour l'étude de leur conductibilité électrique $\sigma$ dans le même intervalle de température. L'absence d'isolation thermique de l'éprouvette dans notre montage expérimental, et la très faible valeur de la conductibilité thermique de cette dernière, de l'ordre de $10^{-2} \mathrm{~W} /{ }^{\circ} \mathrm{C} \mathrm{cm}$, sont la cause d'une inhomogénéité de température au sein de l'éprouvette. En cherchant à mieux connaître la précision de nos mesures de conductibilité électrique $\sigma$ de ces céramiques nous avons pu établir expérimentalement que 
dans un échantillon cylindrique (diamètre $6 \mathrm{~mm}$ et longueur $22 \mathrm{~mm}$ ) la différence de température qui apparaît dans ces conditions entre sa région axiale et sa surface peut être de plusieurs centaines de ${ }^{\circ} \mathrm{C}$. Nous nous sommes proposés d'exploiter cette inhomogénéité de température établie dans le sens radial de l'échantillon pour mesurer sa conductibilité thermique. En effet, la grande importance de cette différence de température nous a permis de penser que cette mesure pouvait être effectuée avec une précision suffisante.

I. Technique expérimentale. - Dans notre dispositif expérimental $\left({ }^{1}\right)$ l'échantillon, en forme d'haltère, est placé entre deux électrodes en platine rhodié $40 \%$. Sa partie centrale est cylindrique. Son diamètre est de $6 \mathrm{~mm}$ et sa longueur de $22 \mathrm{~mm}$. La forme en haltère assure aux extrémités de l'échantillon une température inférieure à celle de sa partie centrale située entre A et B (Fig. 1) qui peut atteindre facilement $2500^{\circ} \mathrm{C}$. Elle protège ainsi dans ce cas les électrodes de la fusion, et par conséquent, permet de conserver des bons contacts électriques entre cellesci et l'éprouvette. Une forme plus simple des échantillons, c'est-à-dire celle d'un bâtonnet cylindrique, est utilisée quand la température de mesure ne dépasse pas $2000^{\circ} \mathrm{C}$. Une légère pression est exercée sur l'échantillon à l'aide d'un ressort. L'action de ce ressort améliore les contacts électriques et compense la dilatation de l'échantillon de céramique à haute température.

Le dégagement de chaleur (effet Joule) provoqué par le passage d'un courant électrique dans une éprouvette de $\mathrm{ZrO}_{2}+\mathrm{CaO}$ ou de $\mathrm{ZrO}_{2}+\mathrm{Y}_{2} \mathrm{O}_{3}$ est directement utilisé pour la porter à haute température $\left(1100-2400^{\circ} \mathrm{C}\right)$. Du fait que ces céramiques ont une résistivité considérable au-dessous de $1000^{\circ} \mathrm{C}$ $\left(\sim 10^{8} \Omega . \mathrm{cm}\right)$ il est indispensable de préchauffer les échantillons afin de pouvoir déclencher leur chauffage par effet Joule. Ce mode de chauffage s'amorce quand la résistance de l'éprouvette devient de l'ordre de $100 \Omega$. Cette valeur de résistance correspond approximativement à $1000^{\circ} \mathrm{C}$ environ pour nos échantillons.

Le préchauffage des échantillons s'effectue à l'aide d'un chalumeau, et ensuite, le chauffage par effet Joule à l'aide d'un générateur de courant alternatif de $3 \mathrm{~kW}$ et de $220 \mathrm{~V}$ de tension de sortie. La puissance dissipée dans l'échantillon varie de 70 à $600 \mathrm{~W} / \mathrm{cm}^{3}$ selon la température qu'on désire obtenir. Sa valeur est déterminée à partir des mesures de la différence de potentiel $\Delta V$ établie entre deux points de l'éprouvette et de l'intensité du courant $I$ qui la traverse. Les mesures électriques sont effectuées par la méthode des quatre points. Deux sondes de prise de potentiel en platine rhodié $40 \%$ sont fixées dans la partie centrale de l'éprouvette (A-B, Fig. 1). La tem-

(1) La mise au point de ce dispositif a été réalisée en collaboration avec M. J. P. Loup du C. N. R. S.

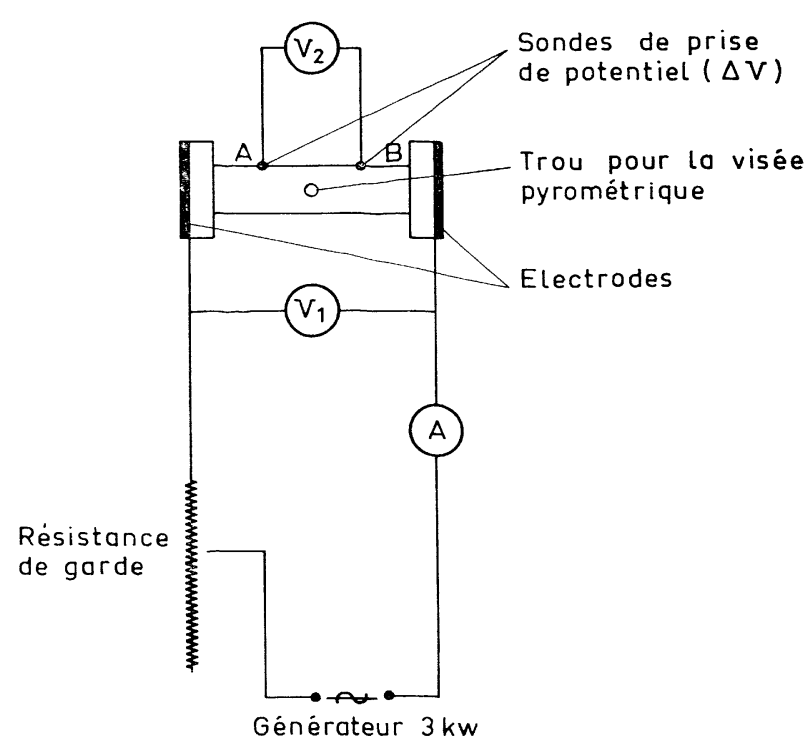

Fig. 1. - Principe du montage de mesure.

pérature de surface $T_{\mathrm{s}}$ est bien homogène dans cette région. La distance qui sépare ces deux sondes est de $1 \mathrm{~cm}$. Ces deux sondes permettent de mesurer la différence de potentiel $\Delta V$ entre deux plans perpendiculaires à l'axe de l'échantillon.

La température $T_{\mathrm{s}}$ en surface de l'échantillon et aussi celle $T_{\mathrm{c}}$ au niveau de sa région axiale sont déterminées par pyrométrie optique. La valeur utilisée pour le pouvoir émissif de la zircone est de 0,35 pour une longueur d'onde de $0,65 \mu$. Nous nous sommes servis d'un pyromètre optique ChauvinArnoux qui permet d'effectuer des visées pyrométriques de champs de très faible surface $\left(\sim 1 \mathrm{~mm}^{2}\right)$. Chaque éprouvette comporte un trou borgne de $1 \mathrm{~mm}$ de diamètre et de $3 \mathrm{~mm}$ de profondeur percé dans la direction radiale. Ce trou est placé entre deux sondes électriques et son rôle est de nous permettre l'exploration de la température $T_{\mathrm{c}}$ au cœur de l'échantillon. La température de surface $T_{\mathrm{s}}$ est déterminée pour plus de précision par la visée de deux champs situés de part et d'autre de ce trou.

Nous n'avons pas étudié le transfert de chaleur dans l'air à partir de la surface de l'échantillon, ni l'importance relative du rayonnement par rapport à la convection. C'est pour s'affranchir de ce problème que nous déterminons expérimentalement la température de surface $T_{\mathrm{s}}$ ainsi d'ailleurs que la température au cœur $T_{\mathrm{c}}$. Evidemment si l'on modifiait l'importance relative du rayonnement par rapport à la convection, les températures $T_{\mathrm{s}}$ et $T_{\mathrm{c}}$ varieraient en conséquence, ce qui reviendrait à mesurer la conductibilité thermique $\lambda$ du matériau à une autre température moyenne $T_{\mathrm{m}}$.

Toutes ces mesures sont entièrement effectuées à l'air libre, ce qui est une des conditions d'utilisation de ces céramiques.

La durée des mesures de la conductibilité thermique 
$\lambda$ et également de la conductibilité électrique $\sigma$ qui sont réalisées simultanément ne dépasse pas une heure pour explorer l'intervalle de température de $1100^{\circ} \mathrm{C}$ à $2500^{\circ} \mathrm{C}$; cette courte durée est due à la très faible inertie thermique des échantillons étudiés et aussi à la technique de leur chauffage par effet Joule.

II. Echantillons étudiés. - Nous avons étudié les échantillons frittés de zircone stabilisée en phase cubique. Cette stabilisation est obtenue par addition à la poudre de $\mathrm{ZrO}_{2}$, soit de $\mathrm{CaO}$, soit de $\mathrm{Y}_{2} \mathrm{O}_{3}$. L'importance des additions de ces oxydes varie de $5 \%$ à $10 \%$. Les échantillons massifs sont ensuite préparés par pressage hydrostatique et frittage à $1600^{\circ} \mathrm{C}$ pendant $12 \mathrm{~h}$ sous hydrogène [1]. La densité de ces zircones se situe entre 5,2 et $5,4 \mathrm{~g} / \mathrm{cm}^{3}$. Les éprouvettes destinées aux mesures sont usinées dans leur forme définitive à l'aide d'outils diamantés.

III. Calcul de la conductibilité thermique. - Rappelons le principe de notre mesure de la conductibilité thermique $\lambda$. Dans l'échantillon cylindrique le chauffage par effet Joule crée une diffusion radiale de chaleur et la différence de température mesurée entre l'axe et la surface est suffisamment grande pour obtenir une valeur précise de la conductibilité thermique du matériau.

Si la puissance était uniformément dissipée suivant le sens radial, la valeur moyenne $\lambda_{0}$ de la conductibilité thermique relative à l'intervalle de température $\left(T_{\mathrm{c}}-T_{\mathrm{s}}\right)$ serait donnée par:

$$
\lambda_{0}=\frac{I \mathrm{~d} V}{4 \pi l\left(T_{\mathrm{c}}-T_{\mathrm{s}}\right)}
$$

$l$ est la hauteur de l'échantillon cylindrique, c'est-àdire la distance en $\mathrm{cm}$ des deux plans perpendiculaires à l'axe entre lesquels est mesurée la différence de potentiel $\mathrm{d} V$ en volts ; $I$ est l'intensité du courant en ampères et $\lambda_{0}$ est exprimée en $\mathrm{W} /{ }^{\circ} \mathrm{C} \mathrm{cm}$.

Dans notre méthode cette hypothèse simple n'est pas valable. Comme le montrera la formulation du problème, la puissance dissipée en un point est fonction de sa température en raison de l'expression de la conductibilité électrique ( $\left.\sigma=A \mathrm{e}^{-E / k T}\right)$, et, en retour, cette température est liée à la répartition de puissance d'après la loi de Fourier.

Nous nous proposons d'effectuer la détermination exacte de la valeur moyenne de $\lambda$ de la conductibilité thermique entre $T_{\mathrm{c}}$ et $T_{\mathrm{s}}$, et de justifier par comparaison le calcul d'un facteur correctif $f_{a}$ qui permet de façon simple d'atteindre une valeur suffisamment approchée de $\lambda$ à partir de $\lambda_{0}$.

La formulation du problème est donnée par l'enchaînement des relations suivantes, où $r$ est la valeur courante du rayon dans une section droite du cylindre. Le rayon extérieur est $a$. La température $T$ est exprimée en ${ }^{\circ} \mathrm{K}$; par simplification nous écrivons $T$ pour $T(r)$ qui désigne la température régnant au rayon $r$.

$$
\lambda \frac{\mathrm{d} T}{\mathrm{~d} r}=-\frac{Q(r)}{2 \pi r l} \quad \text { (loi de Fourier) }
$$

où $Q(r)$ est la puissance totale dissipée dans le sens radial, de l'axe au rayon $r$ :

$$
Q(r)=2 \pi l \int_{0}^{r} q(r) \mathrm{d} r
$$

où $q(r)$ est la puissance spécifique dégagée au rayon $r$ :

$$
q(r)=\frac{\mathrm{d} V}{l} j(r) \quad \text { (loi de Joule) }
$$

où $j(r)$ est la densité de courant électrique au rayon $r$ :

$$
j(r)=\frac{\mathrm{d} V}{l} \sigma(T) \quad(\text { loi d'Ohm })
$$

où $\sigma(T)$ est la conductibilité électrique à la température $T=T(r)$ :

$$
\sigma(T)=A \mathrm{e}^{-E / k T}
$$

où $A$ est un coefficient préexponentiel, $k$ la constante de Boltzmann, $E$ l'énergie d'activation de conduction ; celle-ci est définie par la pente de la courbe expérimentale :

$$
\log \sigma=f(1 / T) .
$$

L'expression de l'intensité du courant électrique

$$
I=2 \pi \int_{0}^{\mathrm{a}} j(r) r \mathrm{~d} r
$$

conduit à la valeur du coefficient préexponentiel :

$$
A=\frac{l}{2 \pi} \frac{I}{\mathrm{~d} V} \cdot \frac{1}{\int_{0}^{\mathrm{a}} r \mathrm{e}^{-\beta / T} \mathrm{~d} r}
$$

avec la notion :

$$
\beta=\frac{E}{k} .
$$

En l'absence de formulation mathématique de la conductibilité thermique en fonction de la température, nous supposons cette grandeur constante dans l'intervalle $\left(T_{\mathrm{c}}-T_{\mathrm{s}}\right)$, et de ce fait nous en déterminons la valeur moyenne $\lambda$.

En intégrant (2) entre l'axe et le rayon $r$, nous égalons l'intégrale de conductibilité, qui est :

$$
\Lambda\left(T, T_{\mathrm{c}}\right)=\lambda\left(T-T_{\mathrm{c}}\right)
$$

à l'intégrale de puissance, ce qui donne :

$$
\Lambda\left(T, T_{\mathrm{c}}\right)=-\frac{I \mathrm{~d} V}{2 \pi i} \frac{\int_{0}^{r} \frac{\mathrm{d} r}{r} \int_{0}^{r} r \mathrm{e}^{-\beta / T} \mathrm{~d} r}{\int_{0}^{\mathrm{a}} r \mathrm{e}^{-\beta / T} \mathrm{~d} r} .
$$


La variable géométrique qui s'impose en raison de la symétrie de révolution est :

$$
u=r^{2} \text {. }
$$

Nous sommes contraints de déterminer simultanément $\lambda$ et la répartition de température, que nous écrivons :

$$
\Theta(u)-T_{\mathrm{c}}=\theta_{1} u+\theta_{2} u^{2}+\cdots+\theta_{n} u^{n}+\cdots
$$

et que nous désignons par la suite $\theta$ des coefficients :

$$
\theta=\left[\theta_{1}, \theta_{2}, \ldots, \theta_{n}, \ldots\right] \text {. }
$$

Définissons les trois séries auxiliaires suivantes :

$$
\begin{aligned}
& S_{0}(u)=\mathrm{e}^{-\beta / \Theta(u)}=s_{0}+s_{1} u+\cdots+s_{n} u^{n}+\cdots \\
& S_{1}(u)=\frac{1}{u} \int_{0}^{u} S_{0}(u) \mathrm{d} u= \\
& \quad=s_{0}+\frac{s_{n}}{2} u+\cdots+\frac{s_{n}}{n+1} u^{n}+\cdots \\
& S_{2}(u)=\frac{1}{u} \int_{0}^{u} S_{1}(u) \mathrm{d} u= \\
& \quad=s_{0}+\frac{s_{1}}{2^{2}} u+\cdots+\frac{s_{n}}{(n+1)^{2}} u^{n}+\cdots
\end{aligned}
$$

L'égalité (4) s'écrit maintenant :

$$
\lambda\left(\Theta(u)-T_{\mathrm{c}}\right)=\Lambda_{0} \frac{u}{a^{2}} \frac{S_{2}(u)}{S_{1}\left(a^{2}\right)},
$$

où $\Lambda_{0}$ est l'intégrale de conductibilité qui correspond à $\lambda_{0}$, c'est-à-dire au cas où la puissance serait uniformément dissipée :

$$
\Lambda_{0}=-\frac{I \mathrm{~d} V}{4 \pi l}
$$

Par identification dans (5) nous avons un premier système de relations entre $\lambda$ et $\theta$ qui traduit la loi Fourier :

$$
\begin{aligned}
& \lambda \theta_{1}=K s_{0} \\
& \lambda \theta_{2}=K \frac{s_{1}}{2^{2}} \\
& \cdots \cdots \cdots \\
& \lambda \theta_{n}=K \frac{s_{n-1}}{n^{2}}
\end{aligned}
$$

où $K$ est une expression qui dépend de la répartition $\theta$

$$
K=\frac{\Lambda_{0}}{a^{2} S_{1}\left(a^{2}\right)} .
$$

Un second système de relations s'obtient en explicitant $S_{0}(u)$. Pour cela nous avons besoin des coefficients de la série :

$$
\begin{aligned}
G(T)=\mathrm{e}^{-\beta / T}=g_{0}+g_{1}\left(T-T_{\mathrm{c}}\right) & +\cdots+ \\
& +g_{n}\left(T-T_{\mathrm{c}}\right)^{n}+\cdots .
\end{aligned}
$$

Grâce aux fonctions intermédiaires :

$$
f^{(0)}=\mathrm{e}^{-\beta / T} \quad \text { et } \quad h^{(0)}=\frac{\beta}{T^{2}}
$$

dont les dérivées à l'ordre $n$ sont :

$$
f^{(n)}=\left[h^{(0)}+f^{(0)}\right]^{(n-1)}
$$

et

$$
h^{(n)}=-\frac{n+1}{T} h^{(n-1)}
$$

nous déterminons de proche en proche le terme général :

$$
g_{n}=f_{\left(T_{c}\right)}^{(n)} / n ! .
$$

Par identification de $S_{0}(u)$ et de $G[\Theta(u)]$ nous obtenons le second système de relations qui traduit l'expression de la conductibilité électrique $\sigma$ en fonction de $\theta$ :

$$
\begin{aligned}
& s_{0}=g_{0} \\
& s_{1}=g_{1} \theta_{1} \\
& s_{2}=g_{2} \theta_{1}^{2}+g_{1} \theta_{2} \\
& s_{3}=g_{3} \theta_{1}^{3}+2 g_{2} \theta_{1} \theta_{2}+g_{1} \theta_{3} .
\end{aligned}
$$

La conductibilité thermique $\lambda$ et la répartition de température $\theta$ doivent vérifier les systèmes (6) et (7) ainsi que la condition :

$$
\Theta\left(a^{2}\right)=T_{\mathrm{s}} .
$$

Leur détermination est obtenue par itération. La convergence est rapide. Les valeurs initiales sont fournies par le cas simple où la puissance est supposée uniformément répartie, c'est-à-dire :

$$
\lambda=\lambda_{0} \quad \text { et } \theta=\theta_{0},
$$

$\theta_{0}$ étant la répartition parabolique de température :

$$
\theta_{0}=\left[\frac{T_{\mathrm{s}}-T_{\mathrm{c}}}{a^{2}}, 0, \ldots, 0, \ldots\right] .
$$

Il résulte de l'équation (5) la valeur moyenne exacte de la conductibilité thermique entre $T_{\mathrm{c}}$ et $T_{\mathrm{s}}$ :

$$
\lambda=\lambda_{0} \frac{S_{2}\left(a^{2}\right)}{S_{1}\left(a^{2}\right)}
$$

et de la relation (3), la valeur exacte du coefficient préexponentiel de la conductibilité électrique $\sigma$ :

$$
A=\frac{l}{\pi} \frac{I}{\mathrm{~d} V} \frac{1}{a^{2} S_{1}\left(a^{2}\right)} .
$$

La conductibilité électrique mesurée dans l'échantillon est :

$$
\sigma_{\mathrm{e}}=\frac{l}{\pi a^{2}} \frac{I}{\mathrm{~d} V} .
$$


Elle nous permet de définir une température équivalente $T_{\mathrm{e}}$ relative à cette mesure de conductibilité électrique :

$$
\sigma_{\mathrm{e}}=A \mathrm{e}^{-\beta / T_{\mathrm{e}}}
$$

ce qui donne d'après (8)

$$
T_{\mathrm{e}}=-\frac{\beta}{\log } \frac{\beta}{S_{1}\left(a^{2}\right)} .
$$

De surcroît, nous obtenons la répartition radiale $\theta$ de la température.

Ce calcul exact, effectué sur ordinateur, montre que la répartition de température s'éloigne assez peu de la répartition parabolique $\theta_{0} ;$ l'écart n'est sensible qu'au voisinage de la surface.

Nous avons cherché une approximation $f_{a}$ du facteur correctif

$$
f=\frac{S_{2}\left(a^{2}\right)}{S_{1}\left(a^{2}\right)}
$$

qui soit d'un emploi commode pour les calculs numériques. Nous en avons éprouvé la validité en comparant $f_{a}$ au calcul exact $f$; l'écart reste inférieur à $1 \%$. Le facteur $f_{a}$ s'obtient en imposant une répartition de température de la forme :

$$
\theta=\left[\theta_{1}, \theta_{2}, 0, \ldots, 0, \ldots\right]
$$

et en limitant le développement de $S_{0}(u)$ aux deux premiers termes.

Ainsi, à partir des valeurs expérimentales déterminées par la méthode que nous proposons, l'obtention numérique de $\lambda$ se réduit à :

$$
\begin{aligned}
\alpha & =\frac{1}{4} \frac{E}{k T_{\mathrm{c}}}\left(1-\frac{T_{\mathrm{s}}}{T_{\mathrm{c}}}\right) \\
f_{\mathrm{a}} & =1+\frac{1}{4}[1+2 \alpha-\sqrt{1-4 \alpha(1+\alpha)]} \\
\lambda_{0} & =\frac{I \mathrm{~d} V}{4 \pi l\left(T_{\mathrm{c}}-T_{\mathrm{s}}\right)} \\
\lambda & =\lambda_{0} \cdot f_{\mathrm{a}} .
\end{aligned}
$$

Le paramètre $\alpha$ qui figure dans l'expression de $f_{a}$ est à la base de la correction que nous nous sommes proposés de trouver. Il réunit les deux caractéristiques physiques du problème : la différence de température $\left(T_{\mathrm{c}}-T_{\mathrm{s}}\right)$ qui permet la mesure de la conductibilité thermique $\lambda$, et, d'autre part, l'argument de l'exponentielle $\left(E / k T_{\mathrm{c}}\right)$ exprimant la variation de la conductibilité électrique $\sigma$ en fonction de la température, grandeur qui à son tour détermine la puissance dissipée.

Nous donnons en figure 2 la variation du facteur correctif $f_{a}$ en fonction du paramètre $\alpha$. En se servant de cette courbe il suffit d'effectuer simplement le calcul de $\alpha$ pour déterminer la valeur de $\lambda$.

Ce développement mathématique nous donne non

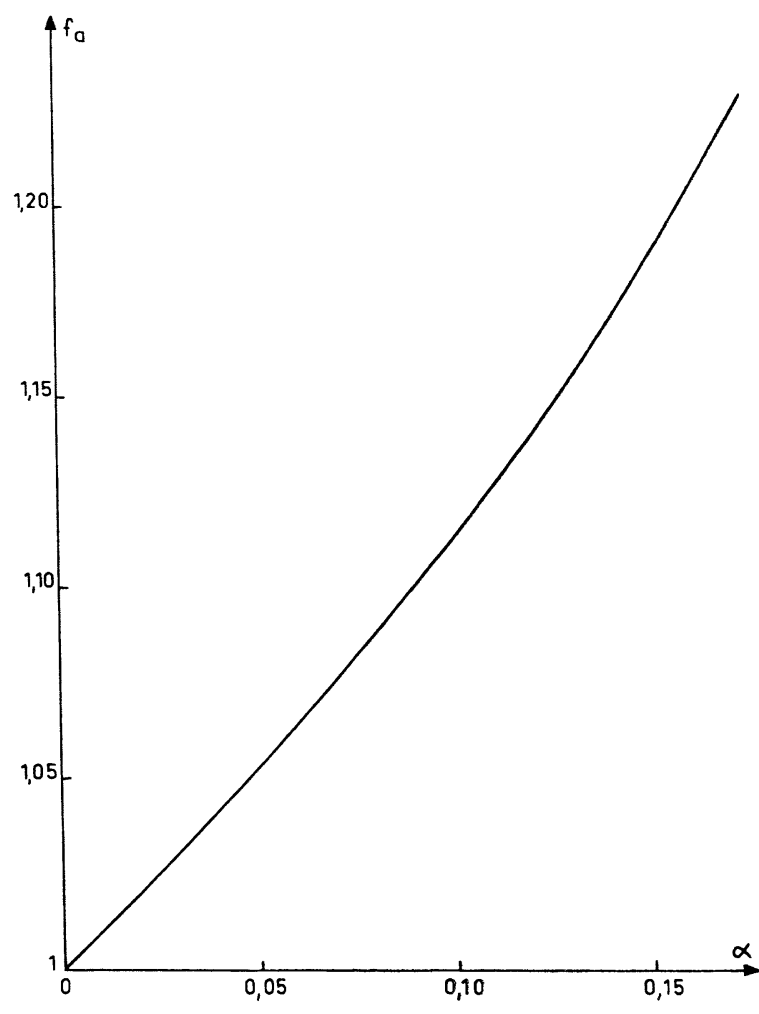

Fig. 2. - Variation du facteur correctif $f_{a}$ en fonction de $\alpha$.

seulement l'expression exacte de $\lambda$, mais également nous permet d'améliorer la précision de nos mesures de conductibilité électrique [1] en nous fournissant une formule plus rigoureuse pour la détermination de la température équivalente $T_{\mathrm{e}}$, c'est-à-dire celle à laquelle correspondent des valeurs mesurées de $\sigma$ quand l'échantillon est chauffé directement par effet Joule. Nous avons obtenu une expression approchée de $T_{\mathrm{e}}$ qui est:

$$
T_{\mathrm{e}}=\frac{T_{\mathrm{c}}+T_{\mathrm{s}}}{2}-0,1 \alpha\left(T_{\mathrm{c}}-T_{\mathrm{s}}\right)
$$

et qui donne une valeur précise du coefficient préexponentiel $\boldsymbol{A}$ dans l'expression de la conductibilité électrique $\sigma$.

$$
A=\frac{l}{\pi a^{2}} \frac{I}{\mathrm{~d} V} \mathrm{e}^{E / k T_{e}} .
$$

La valeur de $A$ ne dépend pas de la température dans un domaine où l'énergie d'activation $E$ est constante. La vérification de cette constance assure la cohérence de cette méthode de mesure.

IV. Résultats expérimentaux. - Les résultats des mesures de la conductibilité thermique $\lambda$ de $\mathrm{ZrO}_{2}+10 \% \mathrm{Y}_{2} \mathrm{O}_{3}$ effectuées dans le domaine de température de $1200^{\circ} \mathrm{C}$ à $2450^{\circ} \mathrm{C}$ sont donnés sur la figure 3. La courbe A représente les valeurs de $\lambda_{0}$, c'est-à-dire celles calculées d'après l'expression (1) qui ne comporte pas le facteur de correction $f_{a}$. Les 


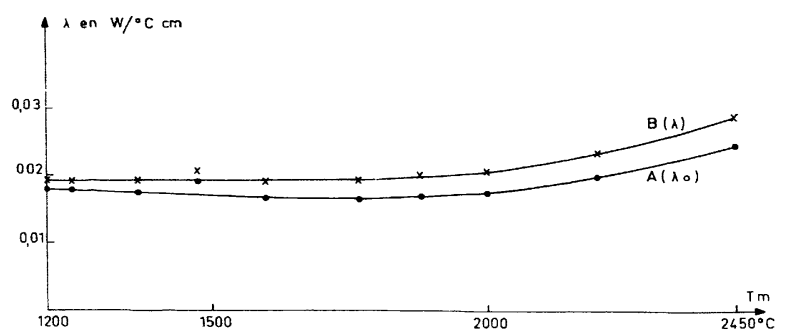

FIG. 3. - Conductibilité thermique de $\mathrm{ZrO}_{2}+10 \% \quad \mathrm{Y}_{2} \mathrm{O}_{3}$ $\left(d=5,4 \mathrm{~g} / \mathrm{cm}^{3}\right)$.

valeurs exactes de $\lambda$ sont données par les points de la courbe $\mathrm{B}$ obtenus par le calcul correctif : $\lambda=\lambda_{0} . f_{a}$. L'énergie d'activation $E$, nécessaire au calcul de $f_{a}$, est déterminée à partir de la pente de la courbe $\log \sigma=f(1 / T)$ résultant des mesures de la conductibilité électrique $\sigma$. Elle a pour valeur $0,52 \mathrm{eV}$ entre 1200 et $1475^{\circ} \mathrm{C}$ et $0,85 \mathrm{eV}$ entre 1600 et $2450 \mathrm{C}^{\circ}$ Le facteur $f_{a}$ n'est pas constant; il varie avec la température. Les valeurs numériques de $\lambda_{0}, f_{a}$ et de $\lambda$ en fonction de la température moyenne $T_{\mathrm{m}}$ sont consignées dans le tableau I.

TABLEAU I

$\begin{array}{cccc}T_{\mathrm{m}} \text { en }{ }^{\circ} \mathrm{C} & \lambda_{\mathrm{o}} \text { en } \mathrm{W} /{ }^{\circ} \mathrm{C} \mathrm{cm} & f_{a} & \lambda \text { en } \mathrm{W} /{ }^{\circ} \mathrm{C} \mathrm{cm} \\ 1 \overline{1} 200 & 0,0181 & 1,06 & 0,0197 \\ 1245 & 0,0180 & 1,06 & 0,0191 \\ 1365 & 0,0175 & 1,074 & 0,0189 \\ 1475 & 0,0192 & 1,075 & 0,0206 \\ 1600 & 0,0164 & 1,147 & 0,0188 \\ 1770 & 0,0159 & 1,214 & 0,0193 \\ 1880 & 0,0168 & 1,203 & 0,0202 \\ 2000 & 0,0173 & 1,201 & 0,0207 \\ 2200 & 0,0200 & 1,190 & 0,0238 \\ 2450 & 0,0248 & 1,172 & 0,0290\end{array}$

Les valeurs de $\lambda$ de $\mathrm{ZrO}_{2}+5 \% \mathrm{CaO}$ sont données sur la figure 4 . Sur cette figure sont aussi représentées les valeurs de $\lambda$ pour cette zircone que nous avons déterminées par la méthode comparative dans l'intervalle de température de $300^{\circ} \mathrm{C}$ à $1150^{\circ} \mathrm{C}$. L'échantillon étalon qui a été utilisé pour ces mesures est en

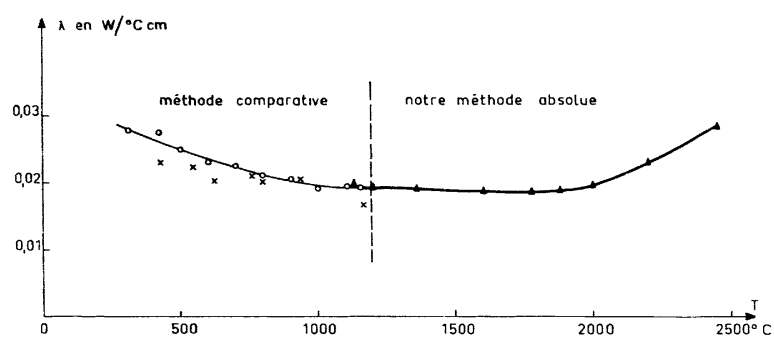

Fig. 4. - Résultats des mesures de la conductibilité thermique de $\mathrm{ZrO}_{2}+5 \% \mathrm{CaO}\left(d=5,2 \mathrm{~g} / \mathrm{cm}^{3}\right)$.

- Méthode comparative : $\bigcirc$ nos mesures

$\times$ mesures de Mirkovich

- Méthode absolue $: \triangle$ nos mesures. alumine frittée. Le raccordement des courbes $\lambda=f\left(T_{\mathrm{m}}\right)$ résultant des mesures par la méthode comparative et la méthode absolue, celle que nous proposons, est très satisfaisant. A titre de comparaison, nous reproduisons aussi sur cette figure les valeurs de $\lambda$ pour une zircone de qualité similaire obtenues par Mirkovich [2] qui a également appliqué la méthode comparative dans ce domaine de température. Nos valeurs de $\lambda$ sont très proches de celles données par cet auteur et dans ces deux cas $\lambda$ évolue dans le même sens avec la température.

Un calcul d'erreur montre que la précision de nos mesures de la conductibilité thermique $\lambda$ est de $\pm 5 \%$ environ. L'incertitude sur la détermination de l'énergie d'activation $E$ n'intervient pas de façon significative sur la précision de $\lambda$.

La correction que $f_{a}$ apporte à $\lambda_{0}$ varie selon la température de $6 \%$ à $20 \%$ environ. Le calcul correctif relève les valeurs de la conductibilité thermique apparente sans influencer d'une manière sensible l'allure générale des courbes $\lambda=f\left(T_{\mathrm{m}}\right)$.

Toutes ces mesures montrent que la conductibilité thermique $\lambda$ de nos échantillons de zircone varie peu entre 1100 et $1900^{\circ} \mathrm{C}$ environ. La valeur moyenne de $\lambda$ dans cet intervalle de température est de

$$
1,9 \times 10^{-2} \mathrm{~W} /{ }^{\circ} \mathrm{C} \mathrm{cm}
$$

ce qui correspond aux bons isolants thermiques. Le transfert de chaleur dans les corps isolants est fondé sur la propagation des ondes d'agitation thermique du réseau appelées phonons.

L'existence du palier sur les courbes $\lambda(T)$ peut s'expliquer en analysant l'expression classique de $\lambda$ obtenue à partir d'un raisonnement analogue à celui de la théorie cinétique des gaz

$$
\lambda=\frac{1}{3} C_{\mathrm{v}} v l .
$$

Cette expression toutefois doit être considérée comme une approximation, mais elle permet la discussion suivante. La chaleur spécifique $C_{\mathrm{v}}$ du corps en question obéit sensiblement à la loi de Dulong et Petit et varie peu à haute température. La vitesse de propagation de phonons $v$ étant déterminée par les constantes élastiques reste pratiquement constante [3]. Le libre parcours moyen $l$ des ondes du réseau est inversement proportionnel à la température $T$, cependant lorsque $T$ croît, $l$, dans un corps polycristallin, ne saurait devenir inférieur à la distance réticulaire moyenne dans la direction de propagation du flux thermique. Nous ne pouvons pas préciser la température à laquelle se produit cette limitation de $l$. Alors deux explications sont possibles pour interpréter l'existence du palier sur les courbes $\lambda(T)$.

$\mathrm{Ou}$ bien à la température où le palier se manifeste la conductibilité thermique par phonons atteint son minimum du fait de la limitation de $l$ par les dimensions des constantes réticulaires.

Ou bien dans le domaine de température du palier $l$ continue à décroître avec $T$ et dans ce cas ce palier 
ne peut s'expliquer que par l'apparition d'un nouveau mécanisme de conduction de chaleur qui compense la diminution de la conductibilité thermique par phonons. De toute façon le relèvement significatif de $\lambda$ au-dessus de $1900^{\circ} \mathrm{C}$ implique l'existence d'un ou de plusieurs mécanismes possibles de transfert calorifique : rayonnement ou déplacement des porteurs de charge électrique ou les deux à la fois. Effectivement, la zircone est un bon conducteur électrique à $2000^{\circ} \mathrm{C}$ $\left(\sigma \simeq 2,5 \Omega^{-1} \mathrm{~cm}^{-1}\right)$.

V. Conclusions. - L'inconvénient de la technique de chauffage direct, c'est-à-dire celle de l'effet Joule, que nous avons d'abord appliquée à la mesure de la conductibilité électrique $\sigma$ de céramiques à haute température, réside, dans nos conditions de travail, dans la différence de température qui s'établit entre le cœur et la surface de l'échantillon. Toutefois, cet inconvénient qui ne permet pas de déterminer avec précision la température à laquelle correspond la valeur mesurée de $\sigma$ a été utilisé avec profit pour mesurer la conductibilité thermique $\lambda$ des mêmes échantillons.
Du fait que dans ce cas la puissance dissipée dans un échantillon n'est pas homogène, un développement mathématique a conduit à la détermination du facteur de correction $f_{a}$ permettant d'atteindre la valeur exacte de la conductibilité thermique $\lambda$. En outre, ce calcul donne la température équivalente $T_{\mathrm{e}}$ dont la connaissance fournit maintenant la précision nécessaire aux mesures de la conductibilité électrique $\sigma$, et de ce fait cette méthode a obtenu l'amélioration indispensable.

Cette méthode utilisant le chauffage direct par effet Joule permet de déterminer simultanément dans un laps de temps d'une heure environ, avec une précision suffisante $( \pm 5 \%$ ) la conductibilité électrique $\sigma$ et la conductibilité thermique $\lambda$ de matériaux réfractaires, bons conducteurs électriques dans un domaine de température allant de $1100^{\circ} \mathrm{C}$ à $2400^{\circ} \mathrm{C}$ et même au-delà si l'état du corps le permet. Ces mêmes mesures réalisées à l'aide d'un four classique demanderaient au moins deux jours de travail.

Nous remercions MM. A. Delaine et P. Morvan pour l'aide apportée à l'exécution des mesures.

\section{Bibliographie}

[1] Elston (J.), Mihailovic (Z.) et Roux (M.), " Electricity from M. H. D. ), Vol. III, p. 389 ; International Atomic Energy Agency Vienna, 1966.

[2] Mirkovich (V. V.), Comparative method and choice of standards for thermal conductivity determinations; J. Am. Ceram. Soc., 1965, 48, 387.

[3] Curien (H.), Séminaire de Chimie Physique, p. 89-97, 1956. 